\title{
Recommandations des Experts
}

Le pouvoir judiciaire constitue l'un des trois pouvoirs de tout Etat démocratique. La qualité de la justice, qui inclut son efficacité, ${ }^{1}$ est une valeur forte de ce pouvoir. Elle est aussi un facteur important de développement économique.

La qualité de la justice peut se résumer ainsi : elle exige une justice indépendante, impartiale et au service des citoyens. Elle suppose aussi un système juridique de qualité.

Après trois ans d'une crise très sévère de la justice et après la réintégration de tous les magistrats révoqués de façon inique, la réforme de la justice serbe s'impose à partir de nouvelles bases. Un processus en ce sens s'est engagé et il convient de le saluer.

Les experts ont participé à des échanges entre différents acteurs de la justice-magistrats et avocats- et des universitaires et ont entendu des représentants du gouvernement serbe ainsi que les ambassadeurs de France et de l'Union Européenne.

Les experts estiment que :

\section{Indépendance et statut de la magistrature}

1. L'indépendance de la Justice serbe doit s'installer de façon pérenne. C'est un élément important pour l'instauration de l'indispensable confiance des citoyens envers l'institution judiciaire. A cet égard, s'impose la redéfinition et la mise en place d'un statut stable de la magistrature ${ }^{2}$ permettant la promotion d'une justice composée de magistrats responsables assumant pleinement l'ensemble de leurs droits et obligations.

2. Dans l'immédiat, il est souhaitable de procéder à de nouvelles élections des représentants des magistrats dans les conseils de justice en raison de la nullité manifeste des élections de 2011 (non-participation au suffrage d'un tiers du corps électoral indûment révoqué).

\section{Calendrier des réformes et identification des urgences}

1. Le calendrier doit être adapté à l'ampleur des réformes en cours (dans tous les champs : pénal, civil, commercial, administratif, organisation judiciaire) et être accompagné d'une étude lucide de leurs conséquences sur les traditions et pratiques juridiques serbes (fondées sur le droit romano-germanique). Mieux vaut prendre le temps d'un approfondissement des objectifs et des modalités de ces réformes, grâce à une évaluation efficace de leur impact et une poursuite de la concertation.

1 Voir définition contenue dans la recommandation CM/Rec. (2010)12 du Comité des Ministres aux Etats membres du Conseil de l'Europe sur les juges : indépendance, efficacité et responsabilités : «L'efficacité consiste à délivrer des décisions de qualité dans un délai raisonnable et sur la base d'une considération équitable des éléments. ».

2 Voir cette recommandation CM/Rec. (2010)12. 
2. Il convient, en outre, d'identifier les dispositions textuelles les plus contestables qui doivent être abolies en urgence.

\section{Concertation, détermination des priorités et des lignes directrices d'une nouvelle justice en Serbie}

1. La concertation doit être élargie par l'implication, selon des modalités à définir, de l'ensemble des magistrats de tous les ressorts judiciaires de la Serbie. Une telle démarche constituerait, en outre, un facteur de cohésion dans un corps judiciaire en recomposition.

2. Dans le cadre de cette concertation, doivent être déterminées les priorités réformatrices en s'appuyant sur la définition de lignes directrices d'une nouvelle justice en Serbie; les modalités de recrutement des magistrats, (qui doivent être les plus objectives possibles) la formation initiale et continue (qui doit être solide, étoffée et adaptée aux besoins) en constituent des composantes très importantes.

\section{Responsabilité disciplinaire}

1. Dans le statut de la magistrature, une attention doit être accordée au champ de la responsabilité disciplinaire, spécialement en prenant en considération les standards européens. Ainsi, sauf malveillance et/ou négligence grossière, les manquements procéduraux ne sauraient, en eux-mêmes, provoquer des poursuites.

2. Spécialement doivent être rapidement remises en cause les dispositions prévoyant des sanctions disciplinaires encourues de plein droit par les juges en cas de non-respect des délais de traitement des dossiers. Des sanctions ne peuvent être envisagées qu'en cas de faute caractérisée d'une gravité avérée. En outre, certains délais prévus par les codes de procédure doivent faire l'objet d'un examen de pertinence dans la perspective d'une nouvelle fixation par le législateur.

\section{$V$. Prise en compte des attentes des citoyens}

Afin de contribuer à la définition des composantes de la qualité de la justice, doit être poursuivie la réflexion sur les attentes des citoyens vis-à-vis de l'institution judiciaire et des magistrats. Il est essentiel de ne pas laisser sans réponse les attentes légitimes.

\section{Qualité du droit}

D’une façon générale, les experts appellent de leur voeu l'installation de la sécurité juridique fondée sur un droit de qualité, dans tous les domaines, cohérent et conforme aux standards européens (CEDH et UE). 
VII. Perspective de réforme constitutionnelle

Enfin, les experts estiment que la Serbie doit s'installer dans la perspective d'une réforme constitutionnelle en vue d'une rénovation structurelle des institutions afin de permettre l'effectivité du respect du principe de la séparation des pouvoirs.

Approuvé par les experts le 28 novembre et (en version définitive) le 4 décembre 2012 ! 